\title{
Correlation between elliptic flow and shear viscosity in intermediate-energy heavy-ion collisions
}

\author{
C. L. Zhou, ${ }^{1}$ Y. G. Ma* ${ }^{*}{ }^{1,2}$ D. Q. Fang, ${ }^{1}$ G. Q. Zhang, ${ }^{1}$ J. Xu, ${ }^{1}$ X. G. Cao, ${ }^{1}$ and W. Q. Shen ${ }^{1,2}$ \\ ${ }^{1}$ Shanghai Institute of Applied Physics, Chinese Academy of Sciences, Shanghai 201800, China \\ ${ }^{2}$ Shanghai Tech University, Shanghai 200031, China
}

(Dated: April 24, 2021)

\begin{abstract}
The correlation between the elliptic flow $v_{2}$ scaled by the impact parameter $b$ and the shear viscosity $\eta$ as well as the specific viscosity $\eta / s$, defined as the ratio of the shear viscosity to the entropy density $s$, is investigated for the first time in intermediate-energy heavy-ion collisions based on an isospin-dependent quantum molecular dynamic model. The elliptic flow is calculated at balance energies to exclude the geometric influence such as the blocking effect from the spectators. Our study shows that $v_{2} / b$ decreases almost linearly with increasing $\eta$, consistent with that observed in ultra-relativistic heavy-ion collisions. On the other hand, $v_{2} / b$ is found to increase with increasing $\eta / s$.

PACS numbers: $25.70 .-\mathrm{z}, 21.65 . \mathrm{Mn}$
\end{abstract}

One of the most important spot in heavy-ion collisions is the phase transition of the strong interacting matter, e.g., the transition between the quark-gluon plasma (QGP) and the hadronic matter at ultra-relativistic energies as well as the liquid-gas phase transition (LGP) at intermediate energies [1 13]. Recently, one interesting probe of the phase transition is the so-called specific viscosity $\eta / s$, defined as the ratio of the shear viscosity $\eta$ to the entropy density $s$. Empirical observations of the temperature or incident energy dependence of $\eta / s$ for $\mathrm{H}_{2} \mathrm{O}, \mathrm{He}$, and $\mathrm{Ne}_{2}$ exhibit a minimum in the vicinity of the phase transition temperature [14]. Besides, with increasing temperature, it is found that $\eta / s$ decreases steeply below and rises slowly above the critical temperature for a wide class of systems. Nevertheless, a lower bound of $\eta / s \geq 1 / 4 \pi$, obtained by Kovtun-Son-Starinets (KSS) for infinitely coupled super-symmetric Yang-Mills gauge theory based on the AdS/CFT duality conjecture, is speculated to be universally valid [15]. It is thus very important to study $\eta / s$ of the strong interacting matter created in heavy-ion collisions at both ultra-relativistic and intermediate energies.

The most common approach to study transport properties of the QGP formed in ultra-relativistic heavyion collisions is to investigate the effects of $\eta / s$ on the elliptic flow in a viscous hydrodynamic model 16 18]. This approach has been implemented to simulate heavy-ion collisions at Relativistic Heavy-Ion Collider (RHIC) and Large Hadron Collider (LHC), and works well in mid-central collisions or at higher energies. In peripheral collisions or at lower energies, however, the hydrodynamic model should be followed by a microscopic transport simulation for the highly dissipative hadronic phase [19, 20]. In addition, the hydrodynamics failed to describe the intermediate-energy heavy-ion collisions that evolve mainly in hadronic degrees of freedom.

*Correspondening author. ygma@sinap.ac.cn
Therefore, no efforts have been devoted to study the viscous effect on the elliptic flow in intermediate-energy heavy-ion collisions. However, some transport models 8 11], e.g., the isospin-dependent quantum molecular dynamic (IQMD) model and the Boltzmann-UehlingUhlenbeck (BUU) model, as well as an isospin- and momentum-dependent interaction [12, 13] can give very reasonable results for intermediate-energy heavy-ion collisions. In our previous studies, efforts have been made to study the LGP through $\eta / s$ based on the IQMD and BUU model, and a good agreement with previous analyses has been found.

It is worth mentioning that the dynamic evolution is more complex in low and intermediate-energy heavy-ion collisions. For instance, the elliptic flow at low energies is related to the rotation of the compound system with the expansion of the hot and compressed participant zone and possibly modified by the shadowing effect of the cold spectator matter. In heavy-ion collisions at ultra-relativistic energies, however, the spectators are quickly separated from the participants, and the participant part can evolve without the influence of spectator matter. It is thus seen that the elliptic flow is positive at low energies, becomes negative at intermediate energies, and is positive again at relativistic energies [21 23]. In transport model simulations at intermediate-energy heavy-ion collisions, nucleon interactions are described by mean fields as well as nucleon-nucleon $(\mathrm{N}-\mathrm{N})$ scatterings in the whole reaction process. With the increasing collision energy, the N-N scatterings become dominating for the dynamics and are responsible for the deflection of the hot compressed participant matter from the cold spectator matter, while the attractive part of the mean field becomes more and more important with decreasing collision energy. These observations indicate that the collective flow of the participant part mainly suffers the compression and pulling out effects from the spectator matter. Therefore, the viscous effect can only be correctly studied once the influence of the spectator on the 
elliptic flow has been successfully removed.

Fortunately, the two competing effects of mean fields and N-N scatterings largely balance each other at the so-called balance energy, characterized by a vanishing direct flow [24 27], i.e., when the slope parameter $F_{d}$ of the directed flow denoting the average transverse momentum in the reaction plane at mid rapidity becomes zero. The influence of the spectator matter on the participant matter is relatively small in collisions at the balance energy, and these collisions provide an excellent opportunity to study the viscous effect on the elliptic flow. One should keep in mind that the referred balance between mean fields and N-N collisions does not mean that they are balanced at the whole collision process. At the maximum compressed stage, the N-N scatterings are stronger while the mean fields take over after some time of expansion. This means that the participant part is still influenced by the spectator matter in the expansion stage. It is thus difficult to calculate reasonably the conventional spatial-eccentricityscaled elliptic flow $v_{2} / \epsilon$ [28, 29], because the spatial eccentricity, which is defined as $\epsilon=\left\langle y^{2}-x^{2}\right\rangle /\left\langle y^{2}+x^{2}\right\rangle$ characterizing the anisotropy in the coordinate space, changes through the whole collision process. Different from that in ultra-relativistic heavy-ion collisions, an impact-parameter-scaled elliptic flow $v_{2} / b$ is introduced to eliminate the geometric effect in the present study.

Our study is based on an isospin-dependent quantum molecular dynamic model (IQMD) [30 32]. In this framework, each nucleon is represented by a Gaussian wave packet in coordinate and momentum space to partially take into account the quantum effects, and the equation of motion for the center of the wave packet evolves according to the classical equation of motion based on the Hamiltonian of the system from effective nucleon interactions. The effective mean field used in the IQMD model can be expressed as 31]

$$
U=U_{\mathrm{Sky}}+U_{\mathrm{Coul}}+U_{\mathrm{Yuk}}+U_{\mathrm{sym}},
$$

where $U_{\text {Sky }}, U_{\text {Coul }}, U_{\text {Yuk }}$, and $U_{\text {sym }}$ are the bulk Skyrme potential, the Coulomb potential, the surface Yukawa potential, and the isospin asymmetry potential, respectively. The bulk Skyrme potential is

$$
U_{\mathrm{Sky}}=\alpha\left(\rho / \rho_{0}\right)+\beta\left(\rho / \rho_{0}\right)^{\gamma},
$$

where $\rho$ is the nucleon number density and $\rho_{0}=0.16$ $\mathrm{fm}^{-3}$ is the saturation density. In this work, the parameters $\alpha=-356 \mathrm{MeV}, \beta=303 \mathrm{MeV}$, and $\gamma=7 / 6$, corresponding to a soft equation of state, are used. The expressions of the other potentials can be found in our previous work [8, 9]. Within the present framework, reasonable phase-space information of nucleons and fragments in intermediate-energy heavy-ion collisions can be obtained. About 50,000 ${ }^{197} \mathrm{Au}+{ }^{197} \mathrm{Au}$ collision events have been simulated using the IQMD model for different impact parameters and beam energies for the investigation of the correlation between the elliptic flow and the shear viscosity in the present study.

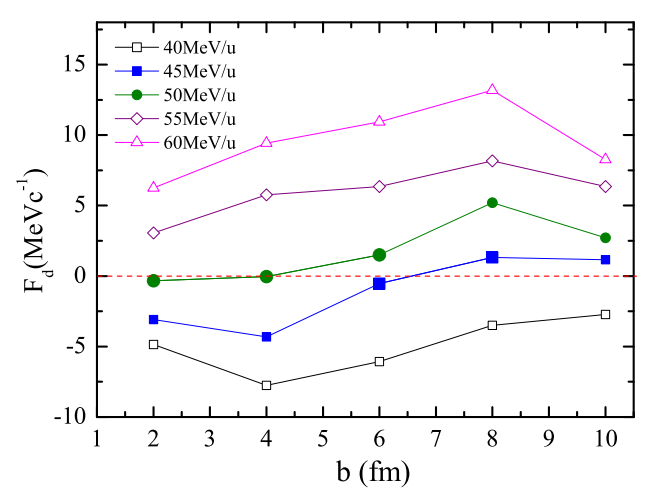

FIG. 1: (Color online) The extracted slope parameter $F_{d}$ of the directed flow as a function of the impact parameter $b$ in heavy-ion collisions at different beam energies.

The slope parameter of the directed flow in non-central heavy-ion collisions can be expressed as

$$
F_{d}=\frac{d\left\langle p_{x} / A\right\rangle}{d\left(y / y_{b}\right)},
$$

where $p_{x}$ is the projection of the transverse momentum in the reaction plane, $A$ is the number of nucleons, and $y / y_{b}$ is the particle rapidity $y$ normalized by the beam rapidity $y_{b}$. The extracted $F_{d}$ from the IQMD model versus the impact parameter $b$ at different beam energies is shown in Fig. 1. The dashed line of $F_{d}=0$ is plotted to guide eyes. With the increase of the impact parameter, it is seen that the $\left|F_{d}\right|$ generally increases, passes through a maximum value, and diminishes in most peripheral collisions 33, 34. Besides, $F_{d}$ is negative at lower energy of $40 \mathrm{MeV} / \mathrm{u}$, positive at higher energies of $55-60 \mathrm{MeV} / \mathrm{u}$, and zero around $45-50 \mathrm{MeV} / \mathrm{u}$, consistent with the findings in Refs. 35 38]. The collision energies and impact parameters with $F_{d}$ most close to 0 are: $[50 \mathrm{MeV} / \mathrm{u}, 2$ fm], [50 MeV/u, $4 \mathrm{fm}$ ], [50 MeV/u, $6 \mathrm{fm}$ ], [45 MeV/u, 6 $\mathrm{fm}]$, and $[45 \mathrm{MeV} / \mathrm{u}, 8 \mathrm{fm}]$, respectively. In these collisions, the effect from the mean field and N-N scatterings cancel each other so that the influence of the blocking from the spectator matter on the elliptic flow are negligible.

For largely equilibrated systems, fluxes of macroscopic quantities are proportional to the field gradient of the system. The shear viscosity $\eta$ is the coefficient of proportionality between the shear force between flow layers per unit area, i.e., the momentum flux, and the velocity gradient, and it can be understood from the fluctuation-dissipation theorem 39]. In the limit of Boltzmann statistics, the shear viscosity corresponds to the first-order ChapmanEnskog coefficient, and can be expressed as a parameterized function by Danielewicz [40, 41]

$$
\begin{aligned}
\eta\left(\frac{\rho}{\rho_{0}}, T\right)= & \frac{1700}{T^{2}}\left(\frac{\rho}{\rho_{0}}\right)^{2}+\frac{22}{1+T^{2} 10^{-3}}\left(\frac{\rho}{\rho_{0}}\right)^{0.7} \\
& +\frac{5.8 \sqrt{T}}{1+160 T^{-2}},
\end{aligned}
$$



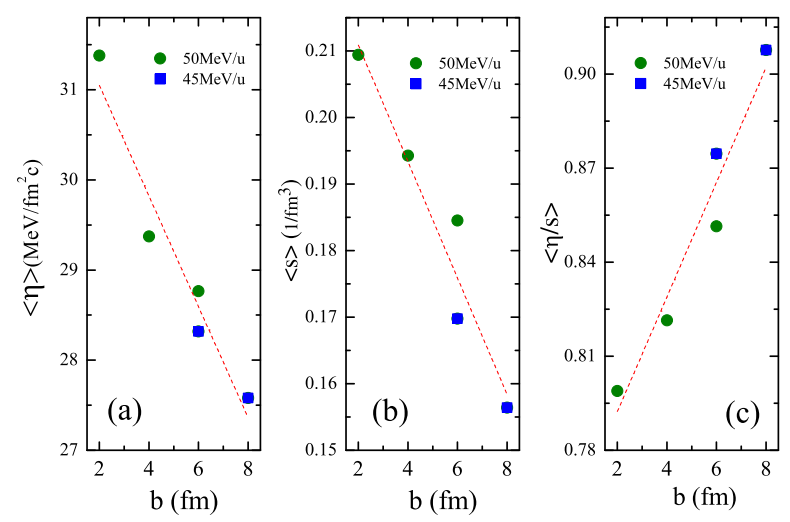

FIG. 2: (Color online) The extracted average shear viscosity (a), entropy density (b), and specific viscosity (c) at balance energies and corresponding impact parameters. The dashed lines are plotted to guide eyes.

where the shear viscosity $\eta$ and the temperature $T$ are in $\mathrm{MeV} / \mathrm{fm}^{2} c$ and $\mathrm{MeV}$, respectively. The above equation is reliable if the system is locally equilibrated with density $\rho$ and temperature $T$, with the former calculated from the overlap of the nucleon wave packets, and the latter as well as the entropy density $s$ obtained from the generalized Thomas-Fermi formulism at finite temperature $42-45]$. The average shear viscosity $\langle\eta\rangle$, entropy density $\langle s\rangle$, and specific viscosity $\langle\eta / s\rangle$ in the center of the system from the moment of maximum compression to freeze-out for the five selected beam energies and impact parameters are given in panel (a), (b), and (c) of Fig. 2. respectively. It is interesting to see that the $\langle\eta\rangle$ decreases with increasing impact parameter. This can be understood from the classical relation $\eta \sim\langle p\rangle / \sigma$, where the average momentum $\langle p\rangle$ is larger in more central collisions while the N-N scattering cross section $\sigma$ is the same. In addition, the $\langle s\rangle$ is also larger in more central collisions due to the higher temperature. After taking the ratio, the average specific viscosity $\langle\eta / s\rangle$ somehow increases with increasing impact parameter.

The elliptic flow is defined as the second-order harmonic coefficient of Fourier expansion of the particle azimuthal distribution

$$
v_{2}=\langle\cos (2 \phi)\rangle=\left\langle\frac{p_{x}^{2}-p_{y}^{2}}{p_{x}^{2}+p_{y}^{2}}\right\rangle,
$$

where $\phi$ is the azimuthal angle, $p_{x}$ and $p_{y}$ are the projections of the transverse momentum parallel and perpendicular to the reaction plane, respectively, and the bracket denotes the average over all the particles. The elliptic flow can be determined by the collective motion resulting from the rotation of the compound system, the expansion of the hot and compressed participant matter, and the possible modification by the shadowing effect of the cold spectator matter [46 51]. Similar to the directed flow, generally the elliptic flow first increases with increasing impact parameter, reaches a maximum in midcentral collisions, and then decreases at large centralities.
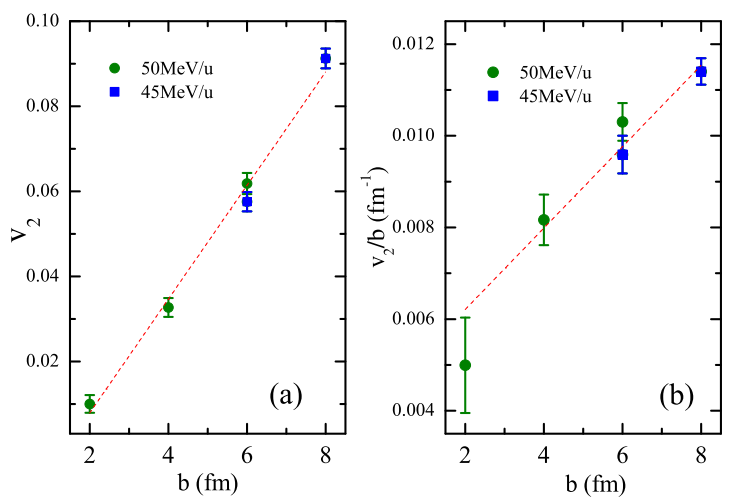

FIG. 3: (Color online) The elliptic flow and that scaled by the impact parameter at balance energies and corresponding impact parameters. The dashed lines are plotted to guide eyes.

In this work the emitted light fragments of charge number $Z \leq 3$ including protons and neutrons at mid-rapidity $\left|y / y_{b}\right| \leq 0.1$ are employed to calculate the elliptic flow at freeze-out. The impact parameter dependence of $v_{2}$ and that scaled by the impact parameter is shown in panel (a) and panel (b) of Fig. 3. respectively. The positive value of $v_{2}$ indicates that an in-plane emission of particles is observed at balance energies. It is shown in panel (a) that $v_{2}$ increases linearly with increasing impact parameter, and the $v_{2}$ at $[45 \mathrm{MeV} / \mathrm{u}, 8 \mathrm{fm}]$ is about 10 times that at $[50 \mathrm{MeV} / \mathrm{u}, 2 \mathrm{fm}]$. After scaled by the impact parameter, this difference is reduced to about 2 times as shown in panel (b), and we argue that the remaining difference is due to the viscous effect in intermediate-energy heavy-ion collisions.

Combing Fig. 2 and Fig. 3, the correlations between $v_{2} / b$ and $\langle\eta\rangle$ as well as $\langle\eta / s\rangle$ are exhibited in panel (a) and (b) of Fig. 4. respectively. It is found that $v_{2} / b$ decreases almost linearly with increasing average shear viscosity $\langle\eta\rangle$. This shows that a stronger interaction, which leads to a smaller shear viscosity, is more efficient in transforming the initial eccentricity to the final elliptic flow, consistent with the findings in heavyion collisions at ultra-relativistic energies. On the other hand, $v_{2} / b$ somehow mostly increases with increasing average specific viscosity $\langle\eta / s\rangle$, different from that in ultrarelativistic heavy-ion collisions. This might be due to the stronger dissipation in the hadronic phase than in the partonic phase, which leads to a different behavior of the entropy density in the former case.

In summary, the correlations between the impactparameter-scaled elliptic flow $v_{2} / b$ and the shear viscosity $\eta$ as well as the specific viscosity $\eta / s$ are investigated based on an isospin-dependent quantum molecular dynamic model. Specific combinations of energy and impact parameter, at which the direct flow disappears, are selected in ${ }^{197} \mathrm{Au}+{ }^{197} \mathrm{Au}$ collisions to remove the blocking effects of the cold spectator matter on the elliptic flow. The shear viscosity is calculated from the parame- 

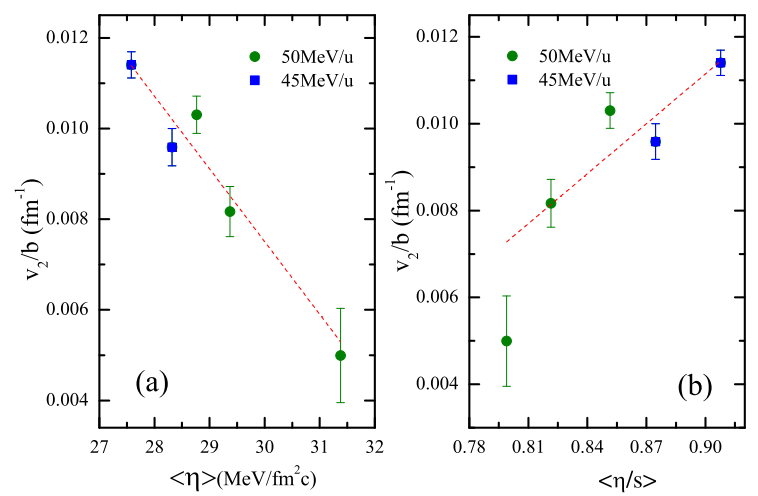

FIG. 4: (Color online) The scaled elliptic flow as a function of average shear viscosity and specific viscosity at balance energies and corresponding impact parameters. The dashed lines are plotted to guide eyes.

terized formulism by Danielewicz for the participant nu- clear matter, and the local density, temperature, and entropy density are extracted from the hot Thomas-Fermi formulism. Our calculation shows the scaled elliptic flow from the light fragments with charge number $Z \leq 3$ decreases within increasing shear viscosity, consistent with that observed in ultra-relativistic heavy-ion collisions at RHIC or LHC. On the other hand, $v_{2} / b$ increases with increasing specific viscosity. Our findings are useful in extracting experimentally the shear viscosity and specific viscosity from the elliptic flow in heavy-ion collisions at balance energies.

This work is partially supported by the NSFC under contracts No.11035009, 11220101005, 10979074, 11175231, 11405248, the Major State Basic Research Development Program in China under Contract No. 2014CB845401, 2013CB834405, the "100-talent plan" of Shanghai Institute of Applied Physics under grant Y290061011 from the Chinese Academy of Sciences, and the Knowledge Innovation Program of Chinese Academy of Science.
[1] Y. G. Ma, Phys. Rev. Lett. 83, 3617 (1999); Y. G. Ma et al., Phys. Rev. C 71, 054606 (2005).

[2] L. G. Moretto, J. B. Elliott, L. Phair, P. T. Lake, J. Phys. G38, 113101 (2011)

[3] J. Adams et al. (STAR Collaboration), Nucl. Phys. A 757, 102 (2005).

[4] R. Lacey et al., Phys. Rev. Lett. 98, 092301 (2007).

[5] G. Giuliani, H. Zheng, A. Bonasera, Prog. Part. Nucl. Phys. 76, 116 (2014); H. Zheng, G. Giuliani, A. Bonasera, Nucl. Sci. Tech. 24, 050512 (2013).

[6] B. Borderie and M. F. Rivet, Prog. Part. Nucl. Phys. 61, 551 (2008).

[7] G. Shao et al., Nucl. Sci. Tech. 24, 050523 (2013); F. M. Liu, Nucl. Sci. Tech. 24, 050524 (2013); C. M. Ko et al., Nucl. Sci. Tech. 24, 050525 (2013).

[8] C. L. Zhou et al., Rhys. Rev. C 88, 024604 (2013).

[9] C. L. Zhou et al., EPL 98, 66003 (2012).

[10] S. X. Li et al., Phys. Rev. C 84, 024607 (2011).

[11] D. Q. Fang, Y. G. Ma, C. L. Zhou, Phys. Rev. C 89, 047601 (2014).

[12] J. Xu et al., Phys. Lett. B 727, 244 (2013).

[13] J. Xu, Nucl. Sci. Tech. 24, 050514 (2013).

[14] L. P. Csernai et al., Phys. Rev. Lett. 97, 152303 (2006).

[15] P. K. Kovtun, D. T. Son, A. O. Starinets, Phys. Rev. Lett. 94, 111601 (2005).

[16] P. Romatschke, U. Romatschke, Phys. Rev. Lett. 99, 172301 (2007).

[17] H. Song, U. W. Heinz, Phys. Rev. C 78, 024902 (2008).

[18] P. Huovinen, D. Molnar, Phys. Rev. C 79, 014906 (2009).

[19] H. Song et al., Phys. Rev. C 83, 024912 (2011).

[20] S. Ryu et al., arXiv: 1210.4588 [hep-ph].

[21] P. Danielewicz et al., Science 298, 1592 (2002).

[22] W. Reisdorf et al., Nucl. Phys. A 876, 1 (2012).

[23] J. Wang et al., Nucl. Sci. Tech. 24, 030501 (2013).
[24] G. F. Bertsch et al., Phys. Lett. B 189, 384 (1987).

[25] D. J. Magestro et al., Phys. Rev. C 61, 021602 (2000).

[26] A. Andronic et al., Phys. Rev. C 64, 041604 (2001).

[27] Rajni et al., Phys. Rev. C 84, 037606 (2011).

[28] G. Ferini et al., Phys. Lett. B 670, 325 (2009).

[29] S. A. Voloshin, A. M. Poskanzerr, Phys. Lett. B 474, 27 (2000).

[30] C. Hartnack et al., Eur. Phys. J. A 1, 151 (1998).

[31] J. Aichelin, Phys. Rep. 202, 233 (1991).

[32] C. Hartnack et al., Nucl. Phys. A 495, 303c (1989).

[33] R. Pak et al., Phys. Rev. C 53, 4 (1996).

[34] S. Soff et al., Phys. Rev. C 51, 6 (1995).

[35] A. D. Sood et al., Phys. Lett. B 594, 260 (2004).

[36] P. Crochet et al., Nucl. Phys. A 624, 755 (1997).

[37] W. M. Zhang et al., Phys. Rev. C 42, 491 (1990).

[38] M. D. Partlan et al., Phys. Rev. Lett. 75, 2100 (1995).

[39] R. Kubo, Rep. Prog. Phys. 29, 255 (1966).

[40] P. Danielewicz, Phys. Lett. B 146, 168 (1984).

[41] B. W. Barker, P. Danielewicz, AIP Conf. Proc. 1231, 167 (2010).

[42] D. T. Khoa et al., Nucl. Phys. A 542, 671 (1992).

[43] P. K. Puri et al., 1992 GSI Scientific Report 93-1, GSI, Darmstadt, Germany, p. 126.

[44] M. Barranco, J. Treiner, Nucl. Phys. A 351, 269 (1981).

[45] M. Rashdan et al., Nucl. Phys. A 468, 168 (1987).

[46] W. K. Wilson et al., Phys. Rev. C 41, 1881 (1990).

[47] M. B. Tsang et al., Phys. Rev. C 47, 2717 (1993).

[48] R. A. Lacey et al., Phys. Rev. Lett. 70, 1224 (1993).

[49] W. K. Wilson et al., Phys. Rev. C 51, 3136 (1995).

[50] Y. G. Ma et al., Phys. Rev. C 48, R1492 (1993); Y. G. Ma et al., Phys. Rev. C 51, 1029 (1995).

[51] Y. M. Zheng et al., Phys.Rev.Lett. 83, 2534 (1999) 